\title{
Uso de medicamentos em crianças menores de um ano
}

\author{
Medication use in children below one-year-old \\ Utilización de medicamentos em niños menores de un año \\ Andressa Larissa Dias Müller de Souza ${ }^{1}$ (D) https://orcid.org/0000-0001-8582-5615 \\ Francieli Ferreira de Andrade Batista ${ }^{1}$ (D) https://orcid.org/0000-0003-1472-3624 \\ Mauren Teresa Grubisich Mendes Tacla ${ }^{1}$ (D) https://orcid.org/0000-0001-8928-3366 \\ Alexandrina Aparecida Maciel Cardelli ${ }^{1}$ (D) https://orcid.org/0000-0002-02228821 \\ Rosângela Aparecida Pimenta Ferrari ${ }^{1}$ (D https://orcid.org/000-0003-0157-7461
}

\section{Resumo}

Objetivo: Analisar o uso de medicamentos em crianças do nascimento ao primeiro ano de vida. Métodos: Estudo descritivo longitudinal, realizada entre 2013 e 2015. Foram realizadas entrevistas com 302 mães em quatro etapas: no pós-parto ainda na maternidade, consulta de retorno puerperal imediato sete dias após 0 parto, visita domiciliar 42 dias pós-parto e visita domiciliar um ano pós-parto.

Resultados: 0 uso de medicamentos nos primeiros 42 dias de vida foi de $76,8 \%$, enquanto no decorrer de um ano, aumentou para 99\%, a automedicação foi realizada em $27,8 \%$ das crianças. Os medicamentos mais utilizados foram paracetamol $(85,1 \%)$, dimeticona $(61,9 \%)$ e antianêmicos $(40,1 \%)$. Os motivos para utilização foram, cólica abdominal, febre, dor, irritabilidade e vacinação.

Conclusão: Foi elevada a incidência do uso de medicamentos, considerando que parte dos sintomas descritos pelas mães podem ser contidos com terapias não farmacológicas. Ressalta-se a necessidade de ações para prevenir o uso indiscriminado de medicamentos para crianças.

\section{Abstract}

Objective: Analyze the medication use in children from birth to the first year.

Methods: Longitudinal descriptive study, conducted between 2013 and 2015. Interviews were carried out with 302 mothers in four stages: maternity postpartum, early puerperal return consultation seven days after birth, household visit 42 days postpartum and household visit one year after postpartum.

Results: Medication use during the first 42 days of life was $76,8 \%$, whereas, in the course of one year, it raised for $99 \%$, the self-medication was performed in $27,8 \%$ of children. The most used medications were paracetamol $(85,1 \%)$, dimethicone $(61,9 \%)$, anti-anemics $(40,1 \%)$. Reasons to use medicines were abdominal colic, fever, pain, irritability, and vaccination.

Conclusion: Incidence of drug utilization was elevated, considering that part of the symptoms described by the mothers could be restrained with non-pharmacological therapies. The necessity of actions to prevent indiscriminate use of drugs for children is highlighted.

\section{Resumen}

Objetivo: Analizar el uso de medicamentos en niños del nacimiento al primer año de vida.

Métodos: Estudio descriptivo longitudinal, realizado entre 2013 y 2015. Se realizaron entrevistas con 302 madres en cuatro etapas: en el post-parto aún en la maternidad, consulta de retorno puerperal inmediato siete días después del parto, visita domiciliar 42 días posparto y visita domiciliar un año después del parto.

Resultados: El uso de medicamentos en niños en los primeros 42 días de vida fue del 76,8\%, mientras que en el transcurso de un año, aumentó al 99\%, la automedicación se realizó en el 27.8\% de los niños. Los medicamentos más utilizados fueron paracetamol $(85,1 \%)$, dimeticona $(61,9 \%)$ y antianémicos $(40,1 \%)$. Los motivos de uso fueron, cólico abdominal, fiebre, dolor, irritabilidad y vacunación.

Conclusión: Fue elevada la incidencia del uso de medicamentos, considerando que parte de los síntomas descritos por las madres pueden ser contenidos con terapias no farmacológicas. Se resalta la necesidad de acciones para prevenir el uso indiscriminado de medicamentos para niños.

\section{Como citar:}

Souza AL, Batista FF, Tacla MT, Cardelli AA, Ferrari RA. [Medication use in children below one-year-old]. Rev Soc Bras Enferm Ped. 2020;20(1):31-9. Portuguese

${ }^{1}$ Universidade Estadual de Londrina, Londrina, PR, Brasil.

Conflitos de interesse: nada a declarar.

Submetido: 7 de Fevereiro de 2020 | Aceito: 1 de Julho de 2020

Autor correspondente: Andressa Larissa Dias Müller de Souza | E-mail: andressadmuller@gmail.com

DOI: http://dx.doi.org/10.31508/1676-3793202000005 


\section{Introdução}

Cotidianamente medicamentos são consumidos de forma indiscriminada, no Brasil a prevalência da automedicação foi de $16,1 \%$, sendo maior na região Nordeste $(23,8 \%) \cdot{ }^{(1)}$ A população tem fácil acesso aos fármacos de venda livre, aqueles sem necessidade de prescrição médica, acarretando em uso irregular e por vezes desnecessárias. O crescimento e propagação da automedicação é um problema de saúde pública no mundo. (2)

Os medicamentos curam e salvam, mas podem também adoecer e matar, sendo que a relação entre a ocorrência de intoxicação ou a cura da doença é a dose utilizada. ${ }^{(3)} \mathrm{A}$ automedicação realizada na população infantil é algo muito comum e preocupante, situações desconfortáveis, como dor, cólica e febre, desencadeiam choro, incitando os pais a realizar este ato, baseando-se na interpretação subjetiva dos sintomas dos seus filhos, no entanto a falta de orientação de um profissional de saúde, acarreta risco a saúde da criança. ${ }^{(4)}$ O uso desnecessário de medicações ocorre corriqueiramente, como por exemplo, instigados pelo medo de uma convulsão febril, muitos pais medicam seus filhos, precocemente, antes que a temperatura atinja $38^{\circ} \mathrm{C}$, mínimo para ser considerado febre. ${ }^{(5,6)}$

Uma pesquisa com 350 crianças de zero a cinco anos revelou que $31,1 \%$ foram automedicadas. ${ }^{(7)} \mathrm{Ou}$ tro estudo europeu realizado com crianças de seis a 11 anos e suas mães, revelou que o uso materno de analgésicos vendidos sem prescrição médica, foi associado significativamente à automedicação entre crianças, com analgésicos, principalmente o paracetamol. Ainda ressaltando sobre a importância do autogerenciamento da dor e outros sintomas inespecíficos para promover o uso apropriado de medicamentos. ${ }^{(8)} \mathrm{O}$ risco do uso indiscriminado de medicamentos pode ser evidenciado na pesquisa sobre internações por intoxicações medicamentosas em crianças menores de cinco anos no Brasil, na qual 6,5\% das intoxicações foram com analgésicos/antitérmicos não opiáceos. ${ }^{(9)}$

A criança difere do adulto por suas características singulares de ordem fisiológica, comportamental e do potencial de exposição a riscos frente às ameaças do ambiente. ${ }^{(10)}$ Uma pesquisa revela que os medicamentos podem ser perigosos para o organismo infantil, visto que, existem poucos estudos de ensaios clínicos que relatem a eficácia e segurança da sua utilização. Razões éticas, legais e econômicas limitam o conhecimento sobre os seus efeitos e, como consequência, o uso de medicamentos em crianças é baseado principalmente em extrapolações e adaptações do uso em adultos. $^{(11)}$

Nos Estados Unidos, existe a Lei Melhores Produtos Farmacêuticos para Crianças e a Lei de Equidade em Pesquisa Pediátrica, que promove ensaios clínicos em populações pediátricas em todas as faixas etárias, realizando a atualização de medicamentos referente à essa população, embora, apesar do progresso, muitos estudos ainda devem ser realizados para promover o desenvolvimento de terapias seguras e eficazes para pacientes pediátricos. ${ }^{(12)}$

Muitos guidelines - diretrizes - para monitoramento e gerenciamento de pacientes pediátricos antes, durante e após a procedimentos terapêuticos, são realizados na tentativa de minimizar os danos, e reduzir os efeitos adversos que os medicamentos podem desencadear nesta população, no entanto, estes guidelines costumam ser direcionados para fármacos utilizados preferencialmente no ambiente hospitalar, ou de uso controlado por prescrição médica, não abrangendo os remédios de venda livre. ${ }^{(13-16)}$

A dose dos medicamentos para população pediátrica é calculada de acordo com o peso ou superfície corporal, idade e quadro clínico. ${ }^{(16,17)}$ Portanto, na automedicação existe probabilidade de ser administrada dose incorreta. Segundo dados fornecidos pelo Sistema Nacional de Informações Tóxico-farmacológicas (SINITOX), em 2013 no Brasil a intoxicação notificada por medicamento foi a maior ocorrência $(29,20 \%)$, sendo $11,74 \%$ entre crianças menores de dez anos de idade. ${ }^{(18)}$ Para tanto, os profissionais de saúde devem orientar sobre medicamentos e os riscos pertinentes da prática da automedicação como medida preventiva, a exemplo nas consultas nas unidades de saúde.

O Ministério da Saúde (MS) recomenda sete consultas de rotina no primeiro ano de vida, duas consultas no $2^{\underline{ }}$ ano de vida e nos anos subsequentes consultas anuais. Essas faixas etárias são selecionadas porque representam momentos de oferta de imunizações e de orientações de promoção de saúde e prevenção de doenças. ${ }^{(19)}$ As consultas de puericultura são necessárias para garantir o crescimento e desenvolvimento saudáveis da criança, também são momentos impor- 
tantes para que o enfermeiro realize orientações sobre os perigos da automedicação, ensinando medidas não farmacológicas de alivio do choro/dor. ${ }^{(20)}$ No entanto, as consultas de puericultura, contém importantes deficiências no processo de trabalho. ${ }^{(21-24)}$

Considerando o primeiro ano de vida, um período de relevante vigilância do desenvolvimento infantil, como também de imaturidade do organismo frente aos efeitos do ambiente, o uso de medicamentos pode oferecer riscos à saúde dessa população. Portanto, o presente estudo teve como objetivo analisar o uso de medicamentos em crianças do nascimento ao primeiro ano de vida, no intuito de identificar a automedicação, os motivos desta prática, bem como os medicamentos mais comumente utilizados.

\section{Métodos}

Estudo descritivo longitudinal, recorte da pesquisa intitulada "Fatores de risco para morbimortalidade materna e infantil: da gestação ao primeiro ano pós-parto", realizada entre julho de 2013 e fevereiro de 2015.

Foi realizado no município de Londrina, Paraná. O local de escolha para a pesquisa foi uma maternidade pública, com atendimento exclusivamente pelo Sistema Único de Saúde e referência para gestação de risco habitual e intermediário que atende $80 \%$ dos partos na cidade, cerca de 300 ao mês.

Utilizando-se da amostragem aleatória simples, a amostra foi calculada considerando o número de 3.415 partos ocorridos na maternidade escolhida, no ano de 2012, considerando margem de erro de 5\% e nível de confiança de $95 \%$, resultando em 358 mulheres. Foram incluídas na amostra mulheres que residiam na zona urbana, ser gestante de risco habitual ou intermediário e ser capaz de compreender e consentir sua participação no estudo. O critério de exclusão foi a mãe encaminhar seu filho para adoção em casa abrigo, pois isso, impossibilita a continuidade da coleta de dados na terceira e quarta etapa, houve apenas uma exclusão, sendo então o $n$ de 357 puérperas.

A pesquisa contemplou 4 etapas para coleta de dados: $1^{a}$. na maternidade em prontuários, Carteira da Gestante e entrevista um dia após o parto; 2aㅡ no ambulatório, em consulta de Retorno Puerperal Imediato 7 a 10 dias após a alta da maternidade; 3ª . Primeira Visita domiciliar (VD) 42 dias após o parto - Retorno Puerperal Tardio (RPT) e; 4a . Segunda VD um ano pós-parto. Mediante aceite e assinatura do Termo de Consentimento Livre e Esclarecido, os dados foram coletados, em formulário previamente testado nesta pesquisa por meio de teste piloto, preenchido por 26 pesquisadores treinados para tal. O contato foi mantido após a alta da maternidade, via telefone para manutenção e atualização de endereço, aceite e desistência da participação na pesquisa. Por meio desse contato foi possível realizar a $4^{a}$ etapa com poucas perdas, sendo que ao final da pesquisa 302 mães participaram das quatro etapas, número total de participantes do presente estudo.

As variáveis de estudo foram: faixa etária materna, situação conjugal, escolaridade, ocupação, classe social, paridade, tipo de parto, peso do recém-nascido, idade gestacional ao nascer, número de consultas de puericultura, uso de medicamentos, classe de medicamentos (antifisético, antiespasmódico, analgésico, antipirético, anti-inflamatório e antianêmico) e orientação por profissional de saúde. Cada classe medicamentosa foi analisada isoladamente considerando a totalidade de crianças, visto que, a mesma criança ingeriu fármacos presentes em mais de uma classe. $\mathrm{O}$ uso de medicamento foi analisado em dois grupos: até 42 dias de vida e 1 ano de idade.

Os dados foram processados e analisados no Statistical Package for the Social Sciences ${ }^{\circledR}$, cruzando frequência de variáveis, aplicando-se o teste qui-quadrado, assumindo um nível de significância de p<0,05 e análises descritivas. O estudo foi aprovado pela Autarquia Municipal de Saúde de Londrina e pelo Comitê de Ética em Pesquisa Envolvendo Seres Humanos da Universidade Estadual de Londrina, Parecer $n^{\circ} .120 .13$ e CAAE: 19352513.9.0000.5231.

\section{Resultados}

Foram acompanhadas nas quatro etapas da pesquisa 302 crianças e suas respectivas mães. Na tabela 1 encontram-se dados sobre as condições socioeconômicas, demográficas, gestacionais, parto, nascimento, assistência no serviço de saúde e uso de medicamentos no primeiro ano de vida. Sendo que, $50,3 \%$ das mães tinham idade $\geq 25$ anos, $85,8 \%$ viviam com companheiro, $75,8 \%$ estudaram 8 anos ou mais, 58,9\% não tinham 
ocupação remunerada e a classe social CD predominou $(82,1 \%)$.

Em relação aos dados obstétricos, 60,3\% eram multíparas, com predominância da via de parto vaginal $(74,8 \%) ; 98 \%$ das crianças pesaram mais do que $2.500 \mathrm{~g}$ ao nascer e com mais de 37 semanas de idade gestacional (97,9\%). Após alta da maternidade, 80,5\% crianças foram acompanhadas no serviço de saúde, participando de cinco consultas ou mais (80,5\%) (Tabela 1$)$.

O uso de medicamentos esteve presente na maioria das crianças (99\%), pois apenas 3 mães relataram não terem medicado seu filho desde o nascimento até um ano de idade. Sendo o paracetamol $(85,1 \%)$, dimeticona $(61,9 \%)$, antianêmicos $(40,1 \%)$ e dipirona sódica $(29,1 \%)$ os mais utilizados. A automedicação foi realizada por $27,8 \%$ das mães e a oferta não foi indicada por profissional de saúde.

A tabela 2 representa os medicamentos comumente utilizados pela mãe do nascimento até um ano de vida. Os medicamentos foram agrupados, unindo várias classes medicamentosas de acordo com a semelhança dos efeitos esperados pelo seu princípio ativo, sendo Classe A: os medicamentos da classe antifisético e antiespasmódico; Classe B: classe de medicamentos analgésicos, antipiréticos e anti-inflamatórios; Classe C: classe de medicamentos antianêmicos.

O uso de medicamentos nos primeiros 42 dias de vida foi de $76,8 \%$. Entre os medicamentos da Classe A, com 42 dias de vida o dimeticona foi o mais utilizado $(44,4 \%)$ e praticamente se manteve até um ano de idade (43\%). Do primeiro grupo (crianças até 42 dias de vida) apenas $35,1 \%$ das mães relatam ter utilizado o medicamento por recomendação de um profissional de saúde, em ambos os grupos as mães utilizaram o medicamento devido ao sintoma de cólica abdominal.

Quanto a Classe B 25,5\% referiu administrar ao seu filho. O paracetamol foi o mais utilizado independentemente da idade, $23,5 \%$ nos primeiros 42 dias de vida e $80,8 \%$ até um ano de idade. A dipirona sódica foi pouco utilizada até 42 dias de vida, apenas em $1 \%$, mas até no primeiro ano totalizou $28,8 \%$. Em ambos os grupos esses medicamentos foram utilizados porque a criança apresentava febre, dor, irritabilidade, também pré e pós vacinação. Aproximadamente $20 \%$ das mães, em ambos os grupos, medicaram por orientação de um profissional de saúde. A classe $\mathrm{C}$ foi a qual mais aumentou, elevando de 0,3\% até 42 dias de vida para
Tabela 1. Condições socioeconômicas, demográficas, gestacionais, parto, nascimento, assistência no serviço de saúde e uso de medicamentos no primeiro ano de vida

\begin{tabular}{|c|c|}
\hline Variáveis & $\begin{array}{c}n(\%) \\
302(100)\end{array}$ \\
\hline \multicolumn{2}{|l|}{ Faixa etária (em anos) } \\
\hline$\leq 24$ & $150(49,7)$ \\
\hline$\geq 25$ & $152(50,3)$ \\
\hline \multicolumn{2}{|l|}{ Situação conjugal } \\
\hline Com companheiro & $259(85,8)$ \\
\hline Sem companheiro & $43(14,2)$ \\
\hline \multicolumn{2}{|l|}{ Escolaridade } \\
\hline$\leq 7$ anos & $73(24,2)$ \\
\hline$\geq 8$ anos & $229(75,8)$ \\
\hline \multicolumn{2}{|l|}{ Ocupação } \\
\hline Remunerada & $124(41,1)$ \\
\hline Não remunerada & $178(58,9)$ \\
\hline \multicolumn{2}{|l|}{ Classe social ${ }^{1}$} \\
\hline$A B$ & $54(17,9)$ \\
\hline$C D$ & $248(82,1)$ \\
\hline \multicolumn{2}{|l|}{ Paridade } \\
\hline Primípara & $120(39,7)$ \\
\hline Multípara & $180(60,3)$ \\
\hline \multicolumn{2}{|l|}{ Tipo de Parto } \\
\hline Vaginal & $226(74,8)$ \\
\hline Cesárea & $76(25,2)$ \\
\hline \multicolumn{2}{|l|}{ Peso do recém-nascido (em gramas) } \\
\hline$<2500$ & $6(2,0)$ \\
\hline$\geq 2500$ & $296(98,0)$ \\
\hline \multicolumn{2}{|l|}{ Idade Gestacional ao Nascer* } \\
\hline$<37$ semanas & $5(2,1)$ \\
\hline$\geq 37$ semanas & $230(97,9)$ \\
\hline \multicolumn{2}{|l|}{ Consultas de Puericultura no serviço de saúde } \\
\hline$\leq 4$ consultas & $59(19,5)$ \\
\hline$\geq 5$ consultas & $243(80,5)$ \\
\hline Ofereceu Medicação & $299(99,0)$ \\
\hline \multicolumn{2}{|l|}{ Medicamentos utilizados } \\
\hline Dimeticona & $187(61,9)$ \\
\hline Paracetamol & $257(85,1)$ \\
\hline Ibuprofeno & $45(14,9)$ \\
\hline Dipirona Sódica & $88(29,1)$ \\
\hline Antianêmico & $121(40,1)$ \\
\hline Uso de medicamento orientado por profissional de saúde & $218(72,2)$ \\
\hline Automedicação & $84(27,8)$ \\
\hline
\end{tabular}

${ }^{1}$ Associação Brasileira de Empresas de Pesquisa (ABEP), ano 2012; *Excluído informações ignoradas

$40,1 \%$ até um ano de idade, predominando a recomendação por um profissional de saúde.

Quanto à faixa etária materna (Tabela 3) os dados são praticamente equivalentes, tanto no primeiro grupo como no segundo. Observou-se que as mães com maior escolaridade referiram medicar mais seus filhos, isso foi evidenciado em todos os tipos de medicamentos ofertados, sendo eles dimeticona, paracetamol, ibuprofeno, dipirona sódica e antianêmico. Con- 
Tabela 2. Medicamentos comumente ofertados pela mãe do nascimento até um ano de vida

\begin{tabular}{lcc}
\hline Variáveis & $\begin{array}{c}42 \text { dias } \\
\mathrm{n}(\%)\end{array}$ & $\begin{array}{c}1 \text { ano } \\
\mathrm{n}(\%)\end{array}$ \\
& $302(100)$ & $302(100)$ \\
\hline Ofereceu medicamento & $232(76,8)$ & $297(98,3)$ \\
Classe A: Antifisético ou antiespasmódico & $134(44,4)$ & $130(43)$ \\
$\quad$ Dimeticona & $134(44,4)$ & $130(43)$ \\
Buscopan ${ }^{\circledR}$ & $2(0,7)$ & $1(0,3)$ \\
$\quad$ Ranitidina & $1(0,3)$ & 0 \\
Motivo da oferta & & \\
$\quad$ Cólica abdominal & $149(81,9)$ & $145(74,4)$ \\
Quem indicou a oferta do medicamento & & \\
$\quad$ Médico ou outro profissional de saúde & $106(35,1)$ & 0 \\
$\quad$ Familiar & $43(14,2)$ & 0 \\
Classe B: Analgésico, antipirético ou anti-inflamatório* & $77(25,5)$ & $288(99,3)$ \\
$\quad$ Paracetamol & $71(23,5)$ & $244(80,8)$ \\
$\quad$ Ibuprofeno & $4(1,3)$ & $44(14,6)$ \\
$\quad$ Dipirona Sódica & $3(1)$ & $87(28,8)$ \\
Motivo da oferta & & \\
$\quad$ Febre, dor, irritabilidade & $40(62,5)$ & $278(92,1)$ \\
$\quad$ Pré e pós vacinação & $17(26,6)$ & $37(12,3)$ \\
Quem indicou a oferta do medicamento & & \\
$\quad$ Médico ou outro profissional de saúde & $61(20,2)$ & $59(19,6)$ \\
$\quad$ Familiar & $12(4)$ & $5(1,7)$ \\
Classe C: Antianêmicos & $1(0,3)$ & $121(40,1)$ \\
Quem indicou a oferta do medicamento & & \\
Médico ou outro profissional de saúde & $1(0,3)$ & $115(38,1)$ \\
\hline
\end{tabular}

forme já mencionado a automedicação considerando a totalidade de crianças ao fim de um ano ocorreu em $27,8 \%$, dentre estas crianças o cruzamento com idade materna e escolaridade, embora não se tenha identificado associação estatisticamente significativa, observou-se que, no decorrer de um ano da criança, as mães mais jovens realizaram com maior frequência a automedicação em seus filhos, e o mesmo foi observado nas mães com maior escolaridade.

Quanto à paridade (Tabela 4), as mães multíparas relataram medicar com maior frequência seus filhos, do que as primíparas, tanto aos 42 dias como até o $1^{0}$ ano de vida. Relacionando-se o número de consultas de puericultura ao longo de um ano, mães que participaram em $\geq 5$ consultas medicaram quatro vezes mais seus filhos, do que as que frequentaram menos, em todas as classes medicamentosas. Ainda o cruzamento entre estas mães, evidenciou que, as que frequentaram no mínimo cinco consultas, realizaram mais a automedicação em seus filhos $(p=0,00)$.

*Excluído informações ignoradas

Tabela 3. Frequência e porcentagem do uso de medicamentos em crianças, distintos nos grupos, 42 dias de vida e um ano de idade, pelas variáveis faixa etária e escolaridade das mães

\begin{tabular}{|c|c|c|c|c|c|c|c|c|c|c|c|c|}
\hline \multirow{3}{*}{ Variáveis } & \multicolumn{6}{|c|}{ Faixa Etária Materna } & \multicolumn{6}{|c|}{ Escolaridade } \\
\hline & \multicolumn{3}{|c|}{42 dias } & \multicolumn{3}{|c|}{1 ano } & \multicolumn{3}{|c|}{42 dias } & \multicolumn{3}{|c|}{1 ano } \\
\hline & $\begin{array}{c}\leq 24 \text { anos } \\
n(\%)\end{array}$ & $\begin{array}{c}\geq 25 \text { anos } \\
n(\%)\end{array}$ & $p$-value & $\begin{array}{c}\leq 24 \text { anos } \\
n(\%)\end{array}$ & $\begin{array}{c}\geq 25 \text { anos } \\
\mathrm{n}(\%)\end{array}$ & p-value & $\begin{array}{c}\leq 7 \text { anos } \\
n(\%)\end{array}$ & $\begin{array}{c}\geq 8 \text { anos } \\
n(\%)\end{array}$ & $p$-value & $\begin{array}{c}\leq 7 \text { anos } \\
\mathrm{n}(\%)\end{array}$ & $\begin{array}{c}\geq 8 \text { anos } \\
\mathrm{n}(\%)\end{array}$ & $p$-value \\
\hline \multicolumn{13}{|l|}{ Medicamento } \\
\hline Ingeriu & $115(49,6)$ & $117(50,4)$ & 0,95 & $148(49,8)$ & $149(50,2)$ & 0,66 & $56(24,1)$ & $176(75,9)$ & 0,98 & $71(23,9)$ & $226(76,1)$ & 0,40 \\
\hline Classe A & $62(46,3)$ & $72(53,7)$ & 0,29 & $63(48,5)$ & $67(51,5)$ & 0,71 & $29(21,6)$ & $105(78,4)$ & 0,35 & $34(26,2)$ & $96(73,8)$ & 0,48 \\
\hline Dimeticona & $62(46,3)$ & $72(53,7)$ & 0,29 & $63(48,5)$ & $67(51,5)$ & 0,71 & $29(21,6)$ & $105(78,4)$ & 0,35 & $34(26,2)$ & $96(73,8)$ & 0,48 \\
\hline Classe B* & $42(54,5)$ & $35(45,5)$ & 0,32 & $144(50,0)$ & $144(50,0)$ & 0,15 & $19(24,7)$ & $58(75,3)$ & 0,90 & $70(24,3)$ & $218(75,7)$ & 0,40 \\
\hline Paracetamol & $39(54,9)$ & $32(45,1)$ & 0,31 & $122(50,0)$ & $122(50,0)$ & 0,81 & $17(23,9)$ & $54(76,1)$ & 0,95 & $57(23,4)$ & $187(76,6)$ & 0,49 \\
\hline Ibuprofeno & $2(50,0)$ & $2(50,0)$ & 0,98 & $21(47,7)$ & $23(52,3)$ & 0,78 & $1(25,0)$ & $3(75,0)$ & 0,96 & $8(18,2)$ & $36(81,8)$ & 0,31 \\
\hline Dipirona Sódica & $1(33,3)$ & $2(66,7)$ & 0,57 & $41(47,1)$ & $46(52,9)$ & 0,57 & $1(33,3)$ & $2(66,7)$ & 0,71 & $25(28,7)$ & $62(71,3)$ & 0,23 \\
\hline Classe C & $0(0,0)$ & $1(100,0)$ & 0,32 & $53(43,8)$ & $68(56,2)$ & 0,09 & $0(0,0)$ & $1(100,0)$ & 0,57 & $27(22,3)$ & $94(77,7)$ & 0,53 \\
\hline Automedicação* & $4(33,3)$ & $8(66,7)$ & 0,06 & $46(54,8)$ & $38(45,2)$ & 0,27 & $2(16,7)$ & $10(83,3)$ & 0,26 & $23(27,4)$ & $61(72,6)$ & 0,41 \\
\hline
\end{tabular}

*Excluído informações ignoradas

Tabela 4. Frequência e porcentagem do uso de medicamentos em crianças, distintos nos grupos, 42 dias de vida e um ano de idade, pelas variáveis paridade e participação em consultas de puericulturas, Londrina, PR, Brasil, 2013-2015.

\begin{tabular}{|c|c|c|c|c|c|c|c|c|c|}
\hline \multirow{3}{*}{ Variáveis } & \multicolumn{6}{|c|}{ Paridade } & \multirow{2}{*}{\multicolumn{3}{|c|}{$\begin{array}{l}\text { Consultas de Puericultura } \\
\qquad 1 \text { ano }\end{array}$}} \\
\hline & \multicolumn{3}{|c|}{42 dias } & \multicolumn{3}{|c|}{1 ano } & & & \\
\hline & $\begin{array}{c}\text { Primípara } \\
\mathrm{n}(\%)\end{array}$ & $\begin{array}{c}\text { Multípara } \\
\mathrm{n}(\%)\end{array}$ & p-value & $\begin{array}{c}\text { Primípara } \\
\mathrm{n}(\%)\end{array}$ & $\begin{array}{c}\text { Multípara } \\
\mathrm{n}(\%)\end{array}$ & p-value & $\begin{array}{c}\leq 4 \\
\mathrm{n}(\%)\end{array}$ & $\begin{array}{c}\geq 5 \\
\mathrm{n}(\%)\end{array}$ & p-value \\
\hline Medicamento & & & & & & & & & \\
\hline Ingeriu & $94(40,5)$ & $138(59,5)$ & 0,61 & $119(40,1)$ & $178(59,9)$ & 0,36 & $59(19,9)$ & $238(80,1)$ & 0,26 \\
\hline Classe A & $55(41,0)$ & $79(59,0)$ & 0,67 & $50(38,5)$ & $80(61,5)$ & 0,69 & $27(20,8)$ & $103(79,2)$ & 0,63 \\
\hline Dimeticona & $55(41,0)$ & $79(59,0)$ & 0,67 & $50(38,5)$ & $80(61,5)$ & 0,69 & $27(20,8)$ & $103(79,2)$ & 0,63 \\
\hline Classe B* & $31(40,3)$ & $46(59,7)$ & 0,91 & $114(39,6)$ & $174(60,4)$ & 0,76 & $56(19,4)$ & $232(80,6)$ & 0,27 \\
\hline Paracetamol & $28(39,4)$ & $43(60,6)$ & 0,95 & $99(40,6)$ & $145(59,4)$ & 0,54 & $46(18,9)$ & $198(81,1)$ & 0,53 \\
\hline Ibuprofeno & $3(75,0)$ & $1(25,0)$ & 0,14 & $23(52,3)$ & $21(47,7)$ & 0,06 & $10(22,7)$ & $34(77,3)$ & 0,56 \\
\hline Dipirona Sódica & $1(33,3)$ & $2(66,7)$ & 0,82 & $28(32,2)$ & $59(67,8)$ & 0,08 & $13(14,9)$ & $74(85,1)$ & 0,20 \\
\hline Classe C & $1(100,0)$ & $0(0,0)$ & 0,21 & $48(39,7)$ & $73(60,3)$ & 0,98 & $16(13,2)$ & $105(86,8)$ & 0,02 \\
\hline Automedicação & $3(25,0)$ & $9(75,0)$ & 0,25 & $34(40,5)$ & $50(59,5)$ & 0,87 & $25(29,8)$ & $59(70,2)$ & 0,00 \\
\hline
\end{tabular}

*Excluído informações ignoradas. 


\section{Discussão}

O uso de medicamentos em menores de um ano de idade conforme a condição socioeconômica encontrada na presente pesquisa revelou-se semelhante aos resultados de estudo que descreveu o perfil de automedicação em crianças de 0 a 5 anos matriculadas em uma creche pública. Os responsáveis tinham escolaridade acima de 7 anos (92\%), 86\% com conjugue e 50\% classe social CD. ${ }^{(25)}$

Foi referido pela maioria das mães neste estudo o uso de medicamentos em seus filhos, sendo que, parte delas realização a automedicação, o mesmo ocorre em outros estudos. ${ }^{(25,26)}$ Quando analisado por etapas, 42 dias de vida e 1 ano, a oferta aumentou ao longo do $1^{\circ}$ ano. Em outras pesquisas tais resultados foram semelhantes, uma na qual $54,8 \%$ dos pais administraram medicamentos para seu filho sem prescrição médica, e em outra, tal dado apresentou $44 \%{ }^{(7,25)}$ Esses achados são preocupantes, visto que o elevado uso de medicações em crianças menores de um ano, pode acarretar riscos para organismo infantil, ressaltando-se a importância das orientações por parte dos enfermeiros pediatras, afim de diminuir esta incidência e fomentar utilização de terapias não farmacológicas para alívio de choro/dor.

Os medicamentos/classes mais utilizados foram os analgésicos, antipiréticos e anti-inflamatórios, os antifiséticos e antiespasmódicos, e os antianêmicos. Contudo, analgésico, antipirético e anti-inflamatório, foram os mais frequentemente ofertados pelas mães, para febre, dor e principalmente antes ou após a vacinação do seu filho, já, no decorrer de um ano, paracetamol, dipirona sódica e ibuprofeno foram os medicamentos mais utilizados, corroborando com resultados de um estudo realizado no estado de Minas Gerais no qual a automedicação ocorreu devido a febre (58\%) e cólica (28\%). ${ }^{(26)}$

Uma pesquisa revela que a prevalência de uso global de medicamentos entre crianças foi de 30,7\%, sendo $5,6 \%$ para doenças crônicas e $27,1 \%$ condições agudas, com maior prevalência de uso pelas crianças menores de dois anos o paracetamol, ácido ascórbico e dipirona. ${ }^{(27)}$ Estudos fazem um alerta sobre o uso indiscriminado de analgésicos e antipiréticos em crianças, refletindo sobre os efeitos adversos. ${ }^{(28)}$

A automedicação é muito mais propícia quando tem-se estoque de medicamentos em domicílio, pois conforme uma pesquisa realizada em Santa Catarina $62,1 \%$ dos entrevistados referiram sobra de tratamentos anteriores, dos quais 52,7\% foram medicamentos pertencentes aos filhos, sendo $21 \%$ analgésicos e antipiréticos. ${ }^{(29)}$ Estudo realizado e Minas Gerais encontraram dados semelhantes, ainda referindo que $27 \%$ dos medicamentos estavam em local de fácil acesso para crianças. ${ }^{(30)}$

Estudos que analisaram prescrições pediátricas destas classes medicamentosas e entrevistas com cuidadores, encontrou a dipirona sódica como fármaco mais frequentemente prescrito $(54,7 \%)$ seguido de ibuprofeno $(26,7 \%)$ e paracetamol $(11,3 \%))^{(31,32)}$ Os resultados também apontaram que $55,4 \%$ das prescrições de dipirona sódica estavam com doses acima das recomendadas por agências reguladoras ou mesmo nas bulas. ${ }^{(31)}$ Neste estudo, somente $20 \%$ da oferta dessa classe medicamentosa foi indicada por um profissional de saúde, ressaltando-se novamente a prática da automedicação. Outra pesquisa também aponta dados semelhantes, relatando sobre a automedicação dos pais nos filhos, utilizando dipirona (54\%) e paracetamol (36\%) em crianças de zero à cinco anos. ${ }^{(26)}$

É possível afirmar o quanto a automedicação implica riscos à saúde infantil, considerando que, mesmo em prescrições médicas a dose do medicamento pode estar acima da recomendada, então, sem o uso de prescrição, é grande a probabilidade da dose administrada estar incorreta. ${ }^{(31)}$ Geralmente a dose para as crianças é pequena, ocasionando perigo na utilização de fármacos sob a forma de comprimidos. ${ }^{(33)}$

Alguns deles apresentam sulco para processo de partição, porém no Brasil não existe normas regulamentadoras que realizam controle de qualidade nos comprimidos destinados à partição, destacando-se que durante o procedimento pode ocorrer esfarelamento e consequentemente alteração na dosagem. Também podem ocorrer outros riscos, como armazenamento incorreto das frações, ou prescrição de dose muito pequena, sendo necessário partir comprimido em mais frações do que a indicada pelo sulco. ${ }^{(33)}$ Ressaltando-se que partição do comprimido é contraindicada, pois a dispersão do princípio ativo não está igualitariamente sob todo comprimido, acarretando durante a partição em administração de dose incorreta.

Com relação à classe dos antifiséticos e antiespasmódicos, a dimeticona foi o medicamento mais 
ofertado no presente estudo, para tratamento da cólica abdominal. Pesquisa que objetivou caracterizar a inter-relação do manejo da cólica infantil por parte das mães e profissionais da Estratégia Saúde da Família, relata que o surgimento da cólica infantil acontece nas primeiras semanas de vida da criança e persiste até o terceiro mês. O reconhecimento desta cólica para as mães estava pautado no choro estridente, excessivo, prolongado após as mamadas, também as "retorcidas" do corpo e rigidez no abdome do filho. Para o alívio do sofrimento da criança fizeram uso de terapias não medicamentosas e medicamentosas, sendo mais utilizado a dimeticona. ${ }^{(34)}$

A presente pesquisa, como em outros estudos, converge para o mesmo achado, pois mais da metade das mães responderam utilizar terapia medicamentosa para tratamento da cólica abdominal em seu filho, sendo o medicamento mais utilizado o dimeticona, como também, em algumas crianças, uso de buscopan ${ }^{\circledR}{ }^{(35,36)}$ Estudo apontou que os sentimentos maternos mais comuns frente a cólica abdominal do seu filho são: tensão, ansiedade, irritação, insegurança, cansaço e depressão, dificultando o manejo para o alívio da dor, impulsionando-as utilizarem medidas farmacológicas. ${ }^{(35)}$

Nesse sentido, é importante ressaltar que, medidas não farmacológicas podem ser mais eficazes para redução da cólica, sendo assim, profissional de saúde, principalmente o enfermeiro pediatra, durante as consultas de puericultura, deve orientar esses métodos, tais como: acalmar o bebê no colo, ou em decúbito ventral, com um "pano morno" ou com o uso de "bolsas térmicas mornas" em contato com o abdômen da criança, como também massagens abdominais. $\mathrm{O}$ uso de sucção não nutritiva pode ser permitido se outras medidas não forem eficazes para alívio do choro/ dor. A técnica de enfaixar o bebê mostra-se eficiente em crianças até 8 semanas, pois aumenta a duração do sono e auxilia na redução da atividade motora. ${ }^{(20,23,37)}$

Outras medidas ainda podem ser utilizadas para alívio das cólicas no lactente, como o uso de probióticos, a redução de alimentos potencialmente alérgenos na dieta materna, para crianças que estiverem em aleitamento materno exclusivo, ou para as crianças com aleitamento complementado a alteração para fórmula hidrolisada ${ }^{(38,39)}$ visto que, ensaios clínicos randomizados controlados não evidenciaram diminuição dos sintomas de cólica utilizando-se simeticona. ${ }^{(40)}$
A classe medicamentosa antianêmica, segundo as recomendações do Ministério da Saúde, deve ser administrada para crianças com idade inferior a 24 meses, pois dificilmente ingerem o suprimento adequado de ferro pela alimentação complementar. Sendo assim, o Programa Nacional de Suplementação de Ferro (PNSF) se destina à profilaxia de anemia ferropriva para crianças de 6 a 24 meses, sendo indicada a dose de $1 \mathrm{mg}$ de ferro elementar por quilo de peso diariamente. ${ }^{(41)}$ Embora este programa esteja instituído desde 2005, evidenciou-se que menos da metade das crianças desta pesquisa receberam essa suplementação.

Sabe-se que é fundamental otimizar os níveis de ferro nos recém-nascidos para apoiar a eritropoiese, o crescimento e o desenvolvimento do cérebro, como também de outros órgãos. ${ }^{(42)}$ Estudos ainda destacam a importância da prevenção da anemia ferropriva, portanto este assunto também deve ser abordado pelos enfermeiro pediatras, orientando os pais sobre a necessidade de ferro ao organismo infantil e as suas principais fontes. ${ }^{(42,43)}$

A oferta de medicamentos às crianças foi elevada nesta pesquisa, mais frequentemente entre mães com maior escolaridade e multíparas. Tais resultados foram semelhantes a outro estudo, que analisou a oferta de fármacos e automedicação pelas mães aos seus filhos em hospital universitário da Espanha. ${ }^{(4)}$

O fato das mães que frequentaram no mínimo cinco consultas de puericultura medicarem mais seus filhos pode ser reflexo do excesso de medicações prescritas desnecessariamente. Por outro lado, essas mães também foram as que mais realizaram a automedicação, indicando a carência de orientação nas consultas quanto aos riscos da oferta medicamentosa. Tal achado corrobora com estudo realizado no estado da Paraíba no qual aponta a necessidade da sensibilização e qualificação profissional permanente para que as consultas de puericultura estejam em consonância com os atributos da atenção Primária em Saúde. ${ }^{(21)}$ Ainda, considerando que $3 / 4$ das crianças com 42 dias de vida já tinha sido medicada, pode-se afirmar que este é um assunto que deveria ser abordado desde o pré-natal.

Ressalta-se a dificuldade para discutir os dados desta pesquisa, visto que, não foi encontrado estudos semelhantes, principalmente ausência de pesquisas que abordem essa temática para crianças de até um ano de idade. Portanto, observa-se a importância de novos estudos que relatem sobre este assunto, para ser 
possível elencar os motivos do uso indiscriminado de fármacos, como também a automedicação pelas mães.

\section{Conclusão}

A maioria das crianças usaram medicamentos, parte das mães praticaram a automedicação. A oferta foi mais frequente entre mães com maior escolaridade, multiparidade e que frequentaram mais consultas de puericultura. Os fármacos mais utilizados foram para os sintomas de febre, dor, choro intenso, cólica abdominal e suplementação de ferro. Considerando que parte destes sintomas podem ser contidos com terapias não medicamentosas, e também os riscos que os fármacos oferecem para o organismo infantil, ressalta-se a necessidade de intensificar ações de prevenção desde o pré-natal para evitar o uso indiscriminado de medicamento. As orientações nas consultas da criança nas unidades de saúde, devem alertar sobre os riscos da automedicação, e também o profissional deve atentar-se para que não ocorra o excesso de medicações prescritas desnecessariamente. Destaca-se que novos estudos devem ser realizados sobre o uso de fármacos em pediatria, como também sobre automedicação pelas mães, afim de propor soluções para essa problemática.

\section{Contribuições}

Souza ALDM, Batista FFA, Tacla MTGM, Cardelli AAM e Ferrari RAP contribuíram com a concepção estudo, análise e interpretação dos dados, redação do artigo, revisão crítica relevante do conteúdo intelectual e aprovação da versão final a ser publicada.

\section{Referências}

1. Arrais PS, Fernandes ME, Pizzol TS, Ramos LR, Mengue SS, Luiza VL, et al. Prevalência da automedicação no Brasil e fatores associados. Rev Saúde Pública. 2016;50(Supl2):111.

2. Bermudez JA, Barros MB. Perfil do acesso e da utilização de medicamentos da população brasileira - contribuições e desafios da PNAUM - Inquérito Domiciliar. Rev Saúde Pública. 2016;50(Supl 2):2s.

3. Oliveira JF, Wagner GA, Romano-Lieber NS, Antunes JL. Tendência da mortalidade por intoxicação medicamentosa entre gêneros e faixas etárias no Estado de São Paulo, Brasil, 1996-2012. Ciênc Saúde Coletiva. 2017;22(10):3381-91.

4. Ortiz MV, Ruiz-Cabello FJ, Uberos J, Ros AF, Ortiz CV, Morales MC, et al. Automedicación, autoprescripción y medicación "por poderes" en pediatría. An Pediatr (Barc). 2017;86(5):264-9.
5. Afonso I, Faria S, Martins S, Silva C, Rocha H, Braga R. Conhecimentos, preocupações e atitudes dos pais perante a febre. Rev Port Med Geral Fam. 2018;34(5):268-72.

6. Piloti PJ, Fragoso A, Duarte BK, Damaceno DG, Marin MJ. Febre em crianças: significado atribuído por responsáveis que procuram serviço de urgência e emergência. Invest Qualitativa em Saúde. 2019;2:985-993.

7. Maniero HK, Martins AA, Melo AC, Paz LP, Schraiber RB, Galato D. Uso de medicamentos em crianças de zero a cinco anos de idade residentes no município de Tubarão, Santa Catarina. Rev Paul Pediatr. 2018;36(4):437-44.

8. Jensen JF, Gottschau M, Siersma VD, Graugaard AH, Holstein BE, Knudsen LE, et al. Association of maternal self-medication and over-the-counter analgesics for children. Pediatrics. 2014;133(2):e291-298.

9. Maior MC, Osorio-de-Castro CG, Andrade CL. Internações por intoxicações medicamentosas em crianças menores de cinco anos no Brasil, 2003-2012. Epidemiol Serv Saúde. 2017;26(4):771-82.

10. Perlroth NH, Branco CW. Current knowledge of environmental exposure in children during the sensitive developmental periods. J Pediatr (Rio J). 2017;93(1):17-27.

11. Moraes CG, Mengue SS, Tavares NU, Dal Pizzol TS. Utilização de medicamentos entre crianças de zero a seis anos: um estudo de base populacional no sul do Brasil. Ciênc Saúde Coletiva. 2013;18(12):3585-93.

12. Wang LA, Cohen-Wolkowiez M, Gonzalez D. Advances in pediatric pharmacology, therapeutics, and toxicology. Adv Pediatr. 2016;63(1):227-54.

13. Coté $\mathrm{CJ}$, Wilson $\mathrm{S}$. Guidelines for monitoring and management of pediatric patients before, during, and after sedation for diagnostic and therapeutic procedures. Pediatrics. 2019;143(6):e20191000

14. Kearns GL, Abdel-Rahman SM, Alander SW, Blowey DL, Leeder JS, Kauffman RE. Developmental pharmacology - drug disposition, action, and therapy in infants and children. N Engl J Med. 2003;349(12):1157-67.

15. Cook C. Acute treatment of pediatric migraine - a review of the updated guidelines. Adv Emerg Nurs J. 2020;42(1):4-12.

16. Rashed AN, Jackson C, Gastine S, Hsia Y, Bielicki J, Standing JF, et al. Pediatric pharmacokinetics of the antibiotics in the access and watch groups of the 2019 WHO model list of essential medicines for children: a systematic review. Expert Rev Clin Pharmacol. 2019;12(12):1099-106.

17. Koumpagioti D, Varounis C, Kletsiou E, Nteli C, Matziou V. Evaluation of the medication process in pediatric patients: a meta-analysis. J Pediatr (Rio J). 2014;90(4):344-55

18. Ministério da Saúde. Fundação Oswaldo Cruz. Sistema Nacional de Informações TóxicoFarmacológicas [Internet]. Rio de Janeiro (RJ): FIOCRUZ, 2019 [cited 2020 Jul 17]. Available from: http://sinitox.icict.fiocruz.br/sites/sinitox.icict.fiocruz.br/files//2013Brasil-tabela7.pdf.

19. Brasil. Ministério da Saúde. Secretaria de Políticas de Saúde. Departamento de Atenção Básica. Saúde da criança: acompanhamento do crescimento e desenvolvimento infantil. Brasília (DF): Ministério da Saúde, 2012.

20. Maciel HI, Costa MF, Costa AC, Marcatto JO, Manzo BF, Bueno M. Medidas farmacológicas e não farmacológicas de controle e tratamento da dor em recém-nascidos. Rev Bras Ter Intensiva. 2019;31(1):21-6.

21. Pedraza DF, Santos IS. Assessment of growth monitoring in child care visits at the family health strategy in two municipalities of Paraíba state, Brazil. Epidemiol Serv Saúde. 2017;26(4):847-55.

22. Monteiro AT, Ferrari RA, Tacla MT, Souza AL. Consulta de enfermagem à criança após alta das maternidades: seguimento na atenção primária. Rev Soc Bras Enferm Ped. 2017;17(1):713.

23. Vieira DS, Soares AR, Nóbrega VM, França JR, Collet N, Reichert AP. Acões implementadas por enfermeiros na consulta de puericultura: revisão integrativa da literatura. Revista Enfermagem Atual In Derme. 2018;86(24):1-24.

24. Vieira DS, Santos NC, Nascimento JA, Collet N, Toso BR, Reichert AP. La práctica del enfermero en la consulta de puericultura en la estratégia saúde da família. Texto Contexto Enferm. 2018;27(4):e4890017.

25. Lima TA, Souza PF, Pereira LL, Godoy MF. Automedicação em crianças matriculadas em creche pública. Arq Ciênc Saúde. 2016;23(4):48-53.

26. Telles Filho PC, Pereira Júnior AC. Automedicação em crianças de zero a cinco anos: fármacos administrados, conhecimentos, motivos e justificativas. Esc Anna Nery. 2013;17(2):291-7. 
27. Dal Pizzol TS, Tavares NU, Bertoldi AD, Farias MR, Arrais PS, Ramos LR, et al. Uso de medicamentos e outros produtos com finalidade terapêutica entre crianças no Brasil. Rev Saúde Pública. 2016;50(Supl 2):12s.

28. Lucas EA, Santos AE, Sodré VR, Veiga ME. A problemática da automedicação na infância. Enfermagem Brasil. 2015;14(2):1-11.

29. Beckhauser GC, Valgas C, Galato D. Perfil do estoque domiciliar de medicamentos em residências com crianças. Rev Ciên Farm Básica Apl. 2012;33(4):583-9.

30. Cruz MJ,Azevedo AB, Bodevan EC,Arújo LU, Santos DF. Estoque doméstico e uso de medicamentos por crianças no Vale do Jequitinhonha, Minas Gerais, Brasil. Saúde Debate. 2017;41(114):836-47.

31. Ferreira TR, Lopes LC. Analysis of analgesic, antipyretic, and nonsteroidal anti-inflammatory drug use in pediatric prescriptions. J Pediatr (Rio J). 2016;92(1):81-7.

32. Chaves CM. Administração de medicamentos orais à criança em Unidade de Acolhimento Institucional: avaliação da prática de enfermagem. Fortaleza. Tese [Dissertação] Universidade Federal do Ceará; 2017.

33. Teixeira MT, Sá-Barreto LC, Silva DL, Cunha-Filho MS. Panorama dos aspectos regulatórios que norteiam a partição de comprimidos. Rev Panam Salud Publica. 2016;39(6):372-7.

34. Abreu FC. Cólica infantil: 0 cuidado de mães e equipes da estratégia de saúde da família. São Carlos. Tese [Dissertação] - Universidade Federal de São Carlos. São Carlos; 2015.
35. Christoffel MM, Silva LR, Silva LR, Ferreira AC, Macedo EC. Cólica do lactente: estudo descritivo das práticas de cuidados maternos para alívio da dor. Rev Enferm UFPE on line. 2013;7(10):5876-82

36. Chinawa JM, Ubesie AC,Adimora GN, Obu HA, Eke CB. Mothers' perception and management of abdominal colic in infants in Enugu, Nigeria. Niger J Clin Pract. 2013;16:169-73.

37. Halpern R, Coelho R. Excessive crying in infants. J Pediatr (Rio J). 2016;92(3 Suppl 1):S405.

38. Johnson JD, Cocker K, Chang E. Infantile colic: recognition and treatment.Am Fam Physician. 2015;92(7):577-82.

39. Sarasu JM, Narang M, Shah D. Infantile colic: an update. Indian Pediatr. 2018;55(11):97987.

40. Garrison MM, Christakis DA. A systematic review of treatments for infant colic. Pediatrics. 2000;106(1 Pt 2):184-90.

41. Brasil. Ministério da Saúde. Caderno de atenção básica. Saúde da criança: aleitamento materno e alimentação complementar. 2 ed. Brasília (DF): Ministério da Saúde, 2015.

42. Kling PJ. Iron nutrition, erythrocytes, and erythropoietin in the NICU: Erythropoietic and neuroprotective effects. Neoreviews. 2020;21(2):e80-e88.

43. Khan L. Anemia in childhood. Pediatr Ann. 2018;47(2):e42-e47. 